\title{
Impaired functionality of HDL in diabetes
}

\author{
Hiroshi Noto
}

Received: 31 October 2011/Published online: 26 November 2011

(C) The Japan Diabetes Society 2011

Patients with type 2 diabetes mellitus (T2DM) remain at higher risk of cardiovascular diseases than nondiabetic subjects even when they have achieved the recommended targets of serum lipids, blood glucose and blood pressure. A growing body of recent investigations suggests that the characteristics of plasma lipoproteins may be altered in such conditions.

It is now accepted that a low concentration of highdensity lipoprotein cholesterol (HDL-C) is the most common lipoprotein abnormality in patients with coronary heart disease (CHD), and the level of HDL-C serves as the strongest prediction of CHD risk [1]: for every $1 \mathrm{mg} / \mathrm{dl}$ increase in HDL-C, the predicted incidence of coronary events decreases by $2 \%$ in men and $3 \%$ in women. Besides, low HDL-C is also associated with unfavorable prognosis in patients with CHD. Even with the most aggressive lowdensity lipoprotein-cholesterol (LDL-C) intervention, two thirds of all cardiovascular events are not prevented. Combination therapy involving the addition of aggressive HDL-based interventions may potentially improve this number. Conventional treatment to raise HDL-C includes lifestyle modifications, exercise, smoking cessation, weight control, moderate alcohol intake, a diet rich in n-3 polyunsaturated fatty acids with limited carbohydrates, niacin, fibrates and statins.

HDL forms a structurally and functionally heterogeneous class of lipoproteins. Most of them contain apolipoprotein

H. Noto $(\square)$

Department of Diabetes and Metabolic Medicine,

Diabetes Research Center,

National Center for Global Health and Medicine,

1-21-1 Toyama, Shinjuku,

Tokyo 162-8655, Japan

e-mail: noto-tky@umin.net (apo) A-I as the most abundant protein constituent of the water-soluble surface. The bulk of HDL is formed by spherical particles containing a core of water-insoluble cholesteryl ester. Apo A-I and apo A-II also have biological actions such as receptor binding and the modulation of several enzymes. In addition to these major apolipoproteins, certain HDL subclasses have enzymes such as lecithincholesterol acyltransferase (LCAT), cholesteryl-estertransfer protein (CETP), phospholipid transfer protein (PLTP), anti-oxidative enzymes or bioactive lipids on the surface.

The well-established classic metabolic pathway of HDL is known as reverse cholesterol transport [2], in which HDL transfers cholesterol from non-hepatic cells to the liver and steroidogenic organs. Reverse cholesterol transport consists of several distinct steps [3] (Fig. 1). HDL is transported to the liver by a direct or indirect pathway. In the indirect pathway, cholesteryl ester in HDL is exchanged for triglycerides in apo B-rich very-low-density lipoprotein (VLDL) and LDL through CETP, with uptake of cholesteryl ester by the liver through LDL receptors. At the same time, HDL becomes enriched with triglycerides, which are hydrolyzed by hepatic triglyceride lipase (HTGL). Epidemiological studies indicated CETP deficiency might be an antiatherogenic state due to HDL-C elevation [4], whereas experimental studies suggest the opposite state [5], and clinical trials of CETP modulation have been hindered [6] because of the complex HDL metabolism and functionality.

Insulin resistance is characterized by low HDL-C and high triglyceride levels. Insulin-resistant fat cells undergo greater breakdown of their stored triglycerides and greater release of free fatty acids into the circulation. Increased fatty acids in the plasma lead to increased fatty acid uptake by the liver where they are synthesized into triglycerides. 


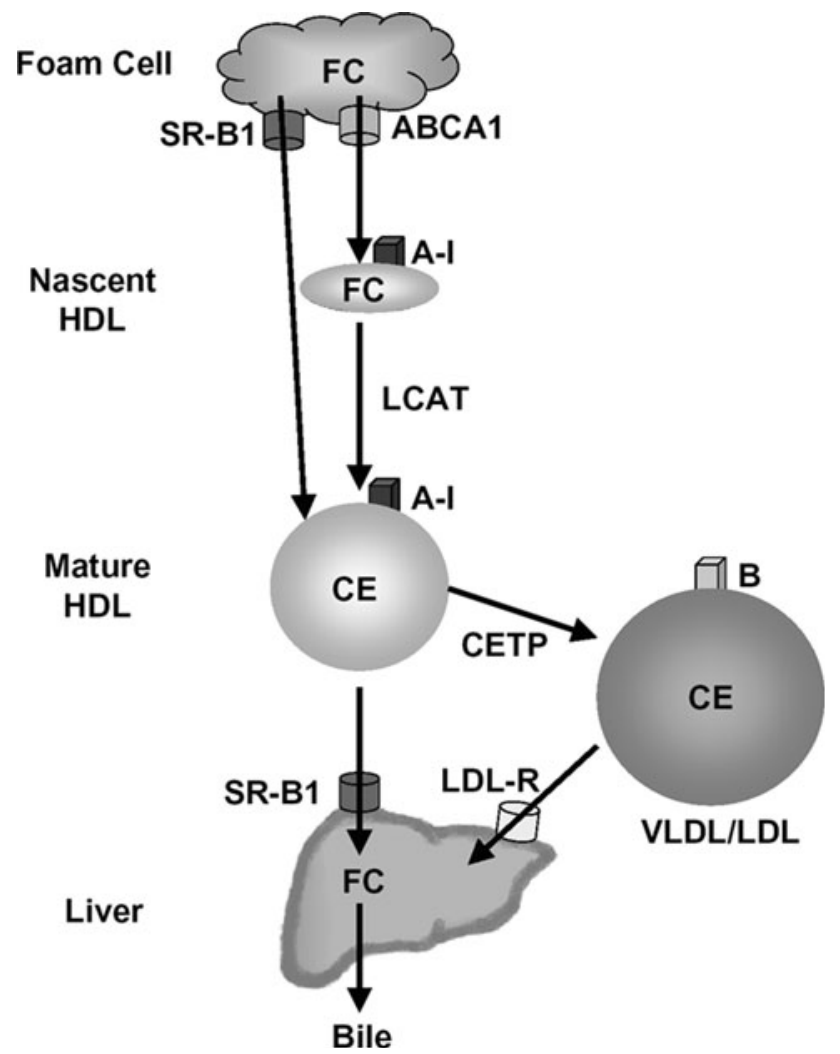

Fig. 1 Reverse cholesterol transport [3]. FC Free cholesterol, SR-B1 scavenger receptor class B type 1, $A B C A 1$ ATP-binding cassette transporter A1, A-I apolipoprotein A-I, $C E$ cholesteryl ester, CETP cholesteryl ester transfer protein, $B$ apolipoprotein $\mathrm{B}, L D L-R$ LDL receptor

The presence of increased triglycerides stimulates the synthesis and secretion of apo B and VLDL, resulting in an increased level of triglycerides in the plasma. In the presence of increased plasma VLDL and normal levels of CETP activity, VLDL triglycerides can be exchanged for HDL-C. This leads to two outcomes: a cholesterol-rich VLDL remnant particle that is atherogenic and a triglyceride-rich cholesterol-depleted HDL particle. The triglyceride-rich HDL particle can undergo further modification including hydrolysis of its triglyceride and dissociation of apo A-I. The free apo A-I in plasma is cleared more rapidly than apo A-I associated with HDL particles. Thus, the amount of circulating apo A-I and the number of HDL particles are reduced. Triglycerides in VLDL are also transferred to LDL in the exchange of cholesteryl ester in LDL. This reaction forms small, dense LDL, which is highly atherogenic.

Atherosclerosis is an inflammatory disorder initiated by an accumulation and subsequent oxidation of LDL in the arterial intima. The oxidized LDL stimulates endothelial cells to express monocyte chemoattractant protein-1 (MCP-1) that, in turn, attracts monocytes into the subendothelial space. Oxidized LDL also promotes the differentiation of monocytes into macrophages that take up the oxidized LDL in a process that converts them into foam cells, the hallmark cell of atherosclerosis. Macrophages also secrete several cytokines that stimulate endothelial cells to express adhesion proteins including vascular cell adhesion molecule-1 (VCAM-1), intercellular adhesion molecule-1 (ICAM-1) and E-selectin. This leads to binding of blood monocytes to the endothelium and its subsequent recruitment. In addition to its function of reverse cholesterol transport, recent investigations have suggested that HDL, as well as its associated proteins, exerts a broad scope of anti-atherogenic functions: (1) antioxidation, (2) antiinflammation, (3) antithrombosis/profibronolysis and (4) endothelial stabilization [3].

In Diabetes, Morgantini et al. [7] reported that antiatherogenic properties of HDL are impaired in T2DM by analyzing the plasma samples from 93 diabetic patients. They first examined the ability of HDL to interfere with LDL-induced monocyte migration in human aortic endothelial cell monolayers. They found the HDL inflammatory index, which is inversely associated with the ability of HDL to facilitate cholesterol efflux and clinically correlated with intima media thickening and atherosclerotic plaque size, was significantly higher than in control subjects $(1.42 \pm 0.29$ in T2DM patients vs. $0.70 \pm 0.19$ in control subjects, $P<0.001)$. In the antioxidation analysis, they observed that the cell-free assay was impaired $(2.03 \pm 1.35$ vs. $1.60 \pm 0.80, P<0.05)$ and HDL intrinsic oxidation was higher $(1,708 \pm 739$ vs. $1,233 \pm 601$ relative fluorescence units, $P<0.001)$ in T2DM, which suggests the antioxidant properties of HDL in T2DM are attenuated. They also found all measured oxidized fatty acids were significantly higher in the HDLs of patients with T2DM. These results support the notion that T2DM is associated with a chronic inflammatory condition. It is speculated that excess generation of reactive oxygen species by hyperglycemia may play a role to impair these functions of HDL. Additionally, in subgroups of patients, ex vivo treatment with L-4F, an apo A-I mimetic peptide that restores HDL function in mice, recovered the HDL inflammatory index in the plasma samples (from $1.26 \pm 0.17$ to $0.71 \pm 0.11, P<0.001$ ). Their findings are clinically meaningful in that they point to the possibility of novel treatment to restore or augment HDL quality.

Several limitations, however, need to be addressed. Because of their study design, it is not possible to determine whether the lipoprotein abnormalities are simply markers or true causes of atherosclerosis. They focused the evaluation on a single facet of the novel function of HDL, and attention needs to be directed to the whole complex process. Inhibition of LDL oxidation by HDL is generally attributed to antioxidative properties of apo A-I, which was shown to reduce peroxides of both phospholipids and cholesteryl esters [8], and to the presence of several enzymes, such as paraoxonase 1 (PON1) [9], platelet- 
activating factor acetylhydrolase (PAF-AH) [10] and glutathione peroxidase, which reportedly prevent the formation or degrade bioactive products of LDL oxidation. There is emerging evidence that $\mathrm{C}$-reactive protein (CRP) may itself contribute to the inflammatory process [11] and HDL inhibits CRP-induced expression of endothelial cell adhesion proteins [12]. Besides, it has been shown that HDL inhibits the expression of cell surface adhesion molecules by activated endothelial cells in vitro [13]. HDL also inhibits the expression of MCP-1 in response to oxidized LDL [14] in a process linked to the antioxidant components of HDL. It is intriguing and critical to explore the state of these interactions in T2DM in further studies.

The finings of Morgantini et al., although preliminary yet, bring us one step higher on the ladder of HDL functionality research, and they underscore the need for better understanding of a mechanism linking inflammation and oxidative stress to the pathogenesis of atherosclerosis in the quest for new agents for the purposes of improving $\mathrm{HDL}$ functionality as well as raising its concentration.

\section{References}

1. Miller M. Raising an isolated low HDL-C level: why, how, and when? Cleve Clin J Med. 2003;70:553-60.

2. Brewer HB Jr. High-density lipoproteins: a new potential therapeutic target for the prevention of cardiovascular disease. Arterioscler Thromb Vasc Biol. 2004;24:387-91.

3. Noto H. Latest insights into high-density lipoprotein functions. The Endocrinologist. 2009;19:179-86.

4. Thompson A, Di Angelantonio E, Sarwar N, et al. Association of cholesteryl ester transfer protein genotypes with CETP mass and activity, lipid levels, and coronary risk. Jama. 2008;299:2777-88.
5. Agerholm-Larsen B, Nordestgaard BG, Steffensen R, et al. Elevated HDL cholesterol is a risk factor for ischemic heart disease in white women when caused by a common mutation in the cholesteryl ester transfer protein gene. Circulation. 2000;101: 1907-12.

6. Stroes ES, van Wijk DF. Dalcetrapib: turning the tide for CETP inhibition? Lancet. 2011;378:1529-30.

7. Morgantini C, Natali A, Boldrini B, et al. Anti-inflammatory and antioxidant properties of HDLs are impaired in Type 2 diabetes. Diabetes. 2011;60:2617-23.

8. Garner B, Waldeck AR, Witting PK, et al. Oxidation of high density lipoproteins. II. Evidence for direct reduction of lipid hydroperoxides by methionine residues of apolipoproteins AI and AII. J Biol Chem. 1998;273:6088-95.

9. Noto H, Hashimoto Y, Satoh H, et al. Exclusive association of paraoxonase 1 with high-density lipoprotein particles in apolipoprotein A-I deficiency. Biochem Biophys Res Commun. 2001; 289:395-401.

10. Noto H, Hara M, Karasawa K, et al. Human plasma plateletactivating factor acetylhydrolase binds to all the murine lipoproteins, conferring protection against oxidative stress. Arterioscler Thromb Vasc Biol. 2003;23:829-35.

11. Pasceri V, Willerson JT, Yeh ET. Direct proinflammatory effect of C-reactive protein on human endothelial cells. Circulation. 2000;102:2165-8.

12. Wadham C, Albanese N, Roberts J, et al. High-density lipoproteins neutralize $\mathrm{C}$-reactive protein proinflammatory activity. Circulation. 2004;109:2116-22.

13. Park SH, Park JH, Kang JS, et al. Involvement of transcription factors in plasma HDL protection against TNF-alpha-induced vascular cell adhesion molecule-1 expression. Int J Biochem Cell Biol. 2003;35:168-82.

14. Navab M, Imes SS, Hama SY, et al. Monocyte transmigration induced by modification of low density lipoprotein in cocultures of human aortic wall cells is due to induction of monocyte chemotactic protein 1 synthesis and is abolished by high density lipoprotein. J Clin Invest. 1991;88:2039-46. 